\title{
Is the standard dose of amoxicillin-clavulanic acid sufficient?
}

\author{
Michiel Haeseker ${ }^{1,3,5^{*}}$, Thomas Havenith², Leo Stolk², Cees Neef ${ }^{2}$, Cathrien Bruggemann ${ }^{1,3}$ and Annelies Verbon ${ }^{4}$
}

\begin{abstract}
Background: The pharmacodynamic (PD) efficacy target of amoxicillin is $40 \%$ time above the minimal inhibition concentration (40\%T > MIC). Recent studies of other antibiotics have shown that PD-efficacy targets are not always reached. The aim of this study was to evaluate the percentage of hospitalised patients, using amoxicillin/clavulanic acid intravenously (iv), that reach the pharmacodynamic efficacy target 40\%T > MIC. Additionally, the association of demographic anthropomorphic and clinical parameters with the pharmacokinetics and pharmacodynamics of amoxicillin were determined.

Methods: In serum of 57 hospitalised patients amoxicillin concentrations were measured using high performance liquid chromatography. Patients were older than 18 years and most patients had an abdominal infection. The standard amoxicillin/clavulanic acid dose was 4 times a day 1000/200 mg iv. Pharmacokinetic parameters were calculated with maximum a posteriori Bayesian estimation (MWPharm 3.60). A one-compartment open model was used. Individual dosing simulations were performed with MMPharm.
\end{abstract}

Results: In our study population, the mean $( \pm \mathrm{SD})$ age was $67( \pm 16)$ years and the mean clearance corrected for bodyweight was $0.17( \pm 0.07) \mathrm{L} / \mathrm{h} / \mathrm{kg}$. Only, 65\% of the patients reached the proposed amoxicillin $40 \% \mathrm{~T}>\mathrm{MIC}$ with amoxicillin/clavulanic acid for bacterial MICs of $8 \mathrm{mg} / \mathrm{L}$. A computer simulated increase of the standard dose to 6 times daily, increased this percentage to $95 \%$. In this small study group $40 \%$ T > MIC was not associated with clinical or microbiological cure.

Conclusion: A substantial proportion of the hospitalised patients did not reach the $40 \% \mathrm{~T}>\mathrm{MIC}$ with the standard dose amoxicillin/clavulanic acid for a bacterial MIC of $8 \mathrm{mg} / \mathrm{L}$. Therefore, we suggest increasing the standard dose of amoxicillin/clavulanic acid to 6 times a day in patients with severe Enterobacteriaceae infections.

Trial registration: Trial registration number: NTR1725 16 ${ }^{\text {th }}$ march 2009.

Keywords: Amoxicillin, Clavulanic acid, Pharmacokinetics, Age

\section{Background}

In vitro and animal studies have shown that $\beta$-lactam antibiotics for Gram-positive bacteria and Gram-negative bacteria are effective when the percentage time above the minimal inhibition concentration $(\% \mathrm{~T}>\mathrm{MIC})$ of the unbound serum concentration is more than $35-40 \%$. Maximal effects are reached with $\% \mathrm{~T}>\mathrm{MIC}$ above $60-70 \%$ [1-3]. Only sparse pharmacokinetic/pharmacodynamic (PK/PD) data are estimated in human clinical studies.

\footnotetext{
*Correspondence: m.haeseker@mumc.nl

'Department of Medical Microbiology, Maastricht University Medical Centre, Maastricht, the Netherlands

${ }^{3}$ Care and Public Health Research Institute (CAPHRI), Maastricht, the Netherlands

Full list of author information is available at the end of the article
}

Pharmacokinetic analysis of amoxicillin/clavulanic acid has mostly been done in healthy individuals $[4,5]$. In human clinical studies amoxicillin/clavulanic acid has been found to cure Streptococcus pneumoniae and Haemophilus influenza infections clinically and microbiologically when $\% \mathrm{~T}>$ MIC was $\geq 40 \%$ [1]. To our knowledge, there are no human pharmacodynamic efficacy studies for Enterobacteriaceae.

Amoxicillin/clavulanic acid is a commonly used broad spectrum antibiotic. Clavulanic acid extends the spectrum of amoxicillin to $\beta$-lactamase producing strains, such as $E$. coli, K. pneumoniae, H. influenzae and S. aureus. Clavulanic acid has very little intrinsic antibacterial effect. Clavulanic acid irreversibly inhibits $\beta$-lactamase, protecting amoxicillin [6]. Therefore, amoxicillin is measured for 
determining the PD-efficacy target. Susceptibility patterns of Enterobacteriaceae have changed over time, and recent studies have shown that efficacy targets of other antibiotics, such as ciprofloxacin [7,8], ceftazidim [9] and gentamicin [10] are not reached in a significant percentage of patients. In the EUCAST rationale document the target attainment rates for different doses of amoxicillin for different MICs based on Monte Carlo simulations are reported [11]. Amoxicillin blood levels have not been measured in these simulations. The aim of this study was to measure the amoxicillin serum concentrations in hospitalised patients using amoxicillin/clavulanic acid intravenously (iv) and to determine if the efficacy target of $40 \% \mathrm{~T}>\mathrm{MIC}$ was reached. Additionally, we have investigated the association of demographic anthropomorphic and clinical parameters with pharmacokinetics and pharmacodynamics of amoxicillin.

\section{Methods}

\section{Study design}

Patients above 18 years of age treated with amoxicillin/clavulanic acid iv and hospitalised at the Maastricht University Medical Centre (MUMC), a 715 bed university hospital, were included from January 2010 until October 2010. Amoxicillin blood levels were measured in residual samples drawn for routine assays. Patients with at least two blood samples available were included and blood samples taken during or within 0.5 hours after infusion were excluded. Amoxicillin/clavulanic acid (Sandoz, Holzkirchen, Germany) was started at the discretion of the attending physician, either empirically or as therapy for bacteria susceptible to amoxicillin/clavulanic acid. The standard dose of amoxicillin/clavulanic acid was 1000/ $200 \mathrm{mg} 4$ times a day and 1000/200 mg 2 times a day was prescribed when the creatinine clearance (CLcr) was 10$50 \mathrm{ml} / \mathrm{min}$. Amoxicillin/clavulanic acid 1000/200 mg/ $100 \mathrm{~mL}$ infusion was infused in 30 minutes. Demographic and clinical data, such as age, gender, weight, temperature, co-medication, length of hospital stay, time of administration of amoxicillin/clavulanic acid and laboratory parameters, such as, serum creatinine, C-reactive protein (CRP) were retrieved from the electronic patient file (iSoft, the Netherlands). Clinical outcome was defined by CRP normalisation, number of admission days, and discharge from the hospital, time to defeveresence and microbiological cure. CLcr was calculated with the Cockcroft-Gault formula using the lean body mass.

This study was registered at the Dutch Trial Register (NTR 1725) [12] and was approved by the Medical Ethical Committee of the Maastricht University Medical Centre (MEC 08-4-063). All data in this study were analysed anonymously and amoxicillin blood levels were measured in residual samples drawn for routine assays. Therefore, no consent was required from the patient.
This is in agreement with the Medical Research Involving Human Subjects Act, the code for proper use of human tissue as formulated by the Dutch Federation of Medical Scientific Societies and the policy of the Medical Ethics Committee of the Maastricht University Medical Centre.

\section{HPLC analysis}

A simple, fast and specific method for measuring amoxicillin serum levels has been validated for linearity, precision, accuracy and stability, following the guidelines for industry bioanalytical method validation recommended by the Food and Drug Administration (FDA) [13]. In short, serum samples were precipitated with perchloric acid 3\%. A reverse phase high pressure liquid chromatography (RP-HPLC) method was used. The calibration range was 10 to $200 \mathrm{mg} / \mathrm{L}$. Six quality controls (8, 20, 40, 60, 80 and $160 \mathrm{mg} / \mathrm{L}$ ) were tested. The intra- and inter-assay variability was within $7.5 \%$. The relationship between plasma concentration and sd was fitted with a polynomial of second order [14]. With this polynomial the lower limit of quantification with a precision of $20 \%$ has been calculated and was $0.8 \mathrm{mg} / \mathrm{L} \mathrm{[15].} \mathrm{All} \mathrm{our} \mathrm{mea-}$ sured concentrations were above $0.8 \mathrm{mg} / \mathrm{L}$.

\section{Pharmacokinetic analysis}

Pharmacokinetic parameters of amoxicillin in individual patients were calculated with maximum a posteriori (MAP) Bayesian estimation program (computer program MW/Pharm 3.60, Mediware, the Netherlands). A onecompartment open pharmacokinetic model was used. With MAP Bayesian estimation [16,17] all patient characteristics and measured amoxicillin concentrations are fitted on an existing population model [4,5]. With two concentrations per patient individual pharmacokinetic parameters can be adequately calculated with MAP Bayesian estimation [17]. With these individual fitted pharmacokinetic parameters, dosing simulations were made to adjust the dose individually; this MAP Bayesian estimation is standard procedure in laboratories which provide therapeutic drug monitoring service. A population kinetic model from our population was not made. The free fraction of amoxicillin was fixed at $80 \%$. The $\% \mathrm{~T}>\mathrm{MIC}$ was determined with the formula of Turnidge; $\% \mathrm{~T}>\mathrm{MIC}=\ln ($ Dose $/ \mathrm{VdxMIC}) \times \mathrm{T}^{1} 12 / \ln (2) \times$ $100 /$ dosing interval [18]. Individual dosing simulations were performed with MW-Pharm (3 times 1000$2000 \mathrm{mg}, 4$ times $2000 \mathrm{mg}, 5$ times 1000-2000 mg, 6 times 1000-2000 mg).

\section{Microbiological analysis}

Identification of the causative bacterium (ID) and antibiotic susceptibility testing (AST) were performed with the Becton Dickinson Phoenix ${ }^{\mathrm{TM}}$ Automated Microbiology 
System (Franklin Lakes, New Jersey, USA) using the ID/ AST Combo panels UNMIC/ID53 and NMIC/ID75 for Gram negative bacteria and the AST panel PMIC-58 for Gram positive bacteria. In urine cultures the lowest MIC detectable was $0.5 \mathrm{mg} / \mathrm{L}$ by the UNMIC/ID53 panel (standard clinical care). MIC values were not determined by the Becton Dickinson Phoenix ${ }^{\text {тM }}$ for Streptococcal spp. and Anaerobes. Susceptibility testing for Streptococcal spp. and Anaerobes was done with the disc diffusion method.

\section{Statistical analysis}

Metric variables were tested for normality of distribution by the Shapiro-Wilk test and presented as mean $( \pm S D)$. If not, median and ranges were also given. Categorical variables are presented as frequencies and percentages. Univariate analysis on the amoxicillin clearance corrected for bodyweight (CLam/W) with categorical variables was either done by Student $t$-test or by one-way ANOVA. Data analysis was done with SPSS-pc version 16.0. A $P$-value of $<0.05$ is considered to be statistically significant.

\section{Results}

Study group

A total of 57 patients with a mean $( \pm S D)$ of $3( \pm 0.9)$ blood samples (median: 2, range 2-5 blood samples) were included. The mean age was $67( \pm 16)$ years (median: 69, range $23-93$ years) and $70 \%$ were male (Table 1$)$. About half of the patients had an abdominal infection, $18 \%$ a wound infection and $10 \%$ a pneumonia (Table 1 ).

\section{Pharmacokinetic and pharmacodynamic analysis}

The mean CLam/W was $0.17( \pm 0.07) \mathrm{L} / \mathrm{h} / \mathrm{kg}$ (median: 0.16 , range: $0.05-0.37 \mathrm{~L} / \mathrm{h} / \mathrm{kg}$ ) and the mean volume of distribution corrected for bodyweight (V/W) was 0.31 $( \pm 0.07) \mathrm{L} / \mathrm{kg}$ (median: 0.30, range: $0.19-0.50 \mathrm{~L} / \mathrm{kg})$. The mean volume of distribution (V) was $24( \pm 5.6) \mathrm{L}$ (median: 24, range: 14-36 L) and the mean elimination half-life $(\mathrm{t} 1 / 2)$ was $1.5( \pm 0.6) \mathrm{h}$ (median: 1.3 , range: 0.6-3.29 h) which is in the same range as described in the EUCAST rationale document [11]. The mean serum creatinine was $90( \pm 36)$ $\mu \mathrm{mol} / \mathrm{L}$ (median: 90, range: 49-210 $\mu \mathrm{mol} / \mathrm{L}$ ). Fifty-five (96\%) patients received the standard dose of amoxicillin/ clavulanic acid 4 times a day 1000/200 mg iv, 1 patient 3 times 1000/200 mg iv and 1 patient 2 times a day 1000/ $200 \mathrm{mg}$ iv, both because of renal insufficiency. Patients above 70 years had lower CLam/W $(P=0.02)$, Table 2 . A significant correlation was found between CLam/W and age $(P<0.001)$.

The measured concentrations of amoxicillin were plotted against the sampling times in Figure 1. There was a good linear correlation $\left(\mathrm{R}^{2}=0.96\right)$ between the amoxicillin actual measured concentration and estimated concentration with MAP Bayesian fitting (MW/Pharm 3.60,
Table 1 Characteristics of $\mathbf{5 7}$ hospitalised patients

\begin{tabular}{|c|c|c|}
\hline & Mean $( \pm S D)$ & Median (range) \\
\hline Age in years & $67( \pm 16)$ & $69(23-93)$ \\
\hline Weight in $\mathrm{kg}$ & $78( \pm 20)$ & $75(43-153)$ \\
\hline Number of blood levels & $3( \pm 0.9)$ & $2(2-5)$ \\
\hline Amoxicillin iv days & $13( \pm 9)$ & $10(3-57)$ \\
\hline Admission days & $27( \pm 34)$ & $19(4-188)$ \\
\hline Gender & Number (percentage) & \\
\hline - Male & $40(70 \%)$ & \\
\hline - Female & 17 (30\%) & \\
\hline Infection & Number (percentage) & \\
\hline - Abdominal infection & $28(49 \%)$ & \\
\hline - Wound infection & $10(17.5 \%)$ & \\
\hline - Pneumonia & $6(11 \%)$ & \\
\hline - Urinary tract infection & $3(5 \%)$ & \\
\hline - Other & $10(17.5 \%)$ & \\
\hline Amoxicillin combination & Number (percentage) & \\
\hline - Monotherapy & $44(78 \%)$ & \\
\hline - Ciprofloxacin & $4(7 \%)$ & \\
\hline - Erythromycin & $3(5 \%)$ & \\
\hline - Gentamicin & $3(5 \%)$ & \\
\hline - Other $^{\mathrm{a}}$ & $3(5 \%)$ & \\
\hline Co-medication & Number (percentage) & \\
\hline - None & $16(28 \%)$ & \\
\hline - Cardiovascular & $27(47 \%)$ & \\
\hline - Diabetic mellitus & $15(26 \%)$ & \\
\hline - Immunosuppressive & $3(5 \%)$ & \\
\hline - Other ${ }^{b}$ & $8(14 \%)$ & \\
\hline
\end{tabular}

ather: rifampicin, cefuroxim and metronidazol.

bOther: haloperidol, levothyroxine, painkillers, anticoagulant.

Mediware, the Netherlands), Figure 2. The amoxicillin efficacy target (40\% T > MIC) was reached in $100 \%$ of patients with a bacterial $\mathrm{MIC} \leq 2 \mathrm{mg} / \mathrm{L}$ of Gram negative bacteria, in $93 \%$ of patients with a $\mathrm{MIC}=4 \mathrm{mg} / \mathrm{L}$ and in $65 \%$ of patients with a MIC $=8 \mathrm{mg} / \mathrm{L}$ (Figure 3 ). When divided in age categories, arbitrarily set at 70 years; all patients older than 70 years reached the $40 \% \mathrm{~T}>\mathrm{MIC}$ with a MIC of $4 \mathrm{mg} / \mathrm{L}$ and $87 \%$ of the patients younger than 70 years. For a MIC of $8 \mathrm{mg} / \mathrm{L}$, the $40 \% \mathrm{~T}>\mathrm{MIC}$ was reached in $81 \%$ of the patients older than 70 years and in $52 \%$ of the patients younger than 70 years (Figure 3).

\section{Analysis of influence of co-variates on pharmacokinetic and pharmacodynamic parameters}

To determine which co-variates have an effect on the pharmacokinetic parameters of amoxicillin, a univariate analysis was done using a predetermined set of predictors (Table 3). In the univariate analysis CLam/W was 
Table 2 Mean $( \pm S D) C L a m / W^{a}, C L C r^{b}, V / W^{c}$ and $T^{1} 2^{d}$ for amoxicillin in patients using amoxicillin/clavulanic acid broken down per age group

\begin{tabular}{llllll}
\hline Age group in years & $\mathbf{N}$ & CLam/W in $\mathbf{L} / \mathbf{h} / \mathbf{k g}$ & $\mathbf{C L c r}$ in $\mathbf{~ m L} / \mathbf{m i n}$ & V/W in L/kg & T1/2 in $\mathbf{h}$ \\
\hline$<70$ & 31 & $0.19( \pm 0.08)$ & $82( \pm 25)$ & $0.30( \pm 0.06)$ & $1.26( \pm 0.44)$ \\
$>70$ & 26 & $0.14( \pm 0.06)$ & $55( \pm 19)$ & $0.32( \pm 0.08)$ & $1.83( \pm 0.71)$ \\
P-value & & 0.02 & $<0.01$ & 0.82 & $<0.01$ \\
\hline
\end{tabular}

${ }^{\mathrm{a}} \mathrm{CLam} / \mathrm{W}$ : amoxicillin clearance corrected for bodyweight.

${ }^{\mathrm{b}} \mathrm{CLCr}$ : creatinine clearance.

V/W: volume of distribution corrected for bodyweight.

${ }^{\mathrm{d}} \mathrm{T} 1 / 2$ : amoxicillin half life.

related to age, creatinine, CLcr, V/W and 40\% $>$ MIC. Linear correlations were found between CLam/W, creatinine and age $\left(R^{2} 0.327\right), \mathrm{Vd}\left(R^{2} 0.370\right)$ and age $\left(R^{2}\right.$ $0.227)$. In the univariate analysis age was not correlated to creatinine $(\mathrm{R} 0.10, P=0.467)$ and no linear correlation $\left(\mathrm{R}^{2}\right.$ 0.01) was found.

\section{Dosing simulations}

To determine whether increasing the dose of amoxicillin/ clavulanic acid would lead to sufficiently high $\% \mathrm{~T}>\mathrm{MIC}$, the $\% \mathrm{~T}>\mathrm{MIC}$ was calculated for all patients with increasing doses with the Turnidge formula [18]. When increasing the dosage frequency from 4 times to 6 times a day all patients with bacterial $\mathrm{MIC} \leq 4$ reach the efficacy target and 95\% (54/57) of the patients with bacterial $\mathrm{MIC} \leq 8$ (Figure 4).

\section{Microbiological analysis}

Sixteen out of twenty one abdominal fluid cultures became positive, $8 / 10$ wound cultures, $7 / 16$ blood cultures, $4 / 5$ urine cultures and $1 / 2$ sputum cultures became positive. Of three patients no cultures were taken. In total thirtysix of the 57 patients $(63 \%)$ had a positive culture. Of the positive cultures, 30 (83\%) were Enterobacteriaceae; E. coli $(\mathrm{n}=21)$, Klebsiella spp. $(\mathrm{n}=6)$, Enterobacter $\mathrm{spp}$. $(\mathrm{n}=2)$ and Proteus spp. $(\mathrm{n}=1)$. In three patients two bacteria were isolated. Of the Gram positive cultures, 6 were Enterococcus spp, 3 Staphylococcus aureus and 1 coagulase negative staphylococcus. Forty MIC values were available in 36 patients. Of the isolated Enterobactericeae $17 / 30(57 \%)$ of had a MIC $\leq 4 \mathrm{mg} / \mathrm{L}, 4 / 30(13 \%)$ had a MIC $=8 \mathrm{mg} / \mathrm{L}$ and $9 / 30(30 \%)$ had a $\mathrm{MIC} \geq$ $16 \mathrm{mg} / \mathrm{L}$. Of the isolated Gram positive bacteria 8/10 $(80 \%)$ of had a MIC $\leq 1 \mathrm{mg} / \mathrm{L}$ and $2 / 10(20 \%)$ had a $\mathrm{MIC} \geq 4 \mathrm{mg} / \mathrm{L}$. Clinical cure was reached in 46 patients, 8 patients switched antibiotic therapy and in 3 patients cure was not reached (of which two died). No significant associations were found between $40 \% \mathrm{~T}>\mathrm{MIC}$ and defeverescence, CRP decrease or increase, admission days and clinical outcome (data not shown).

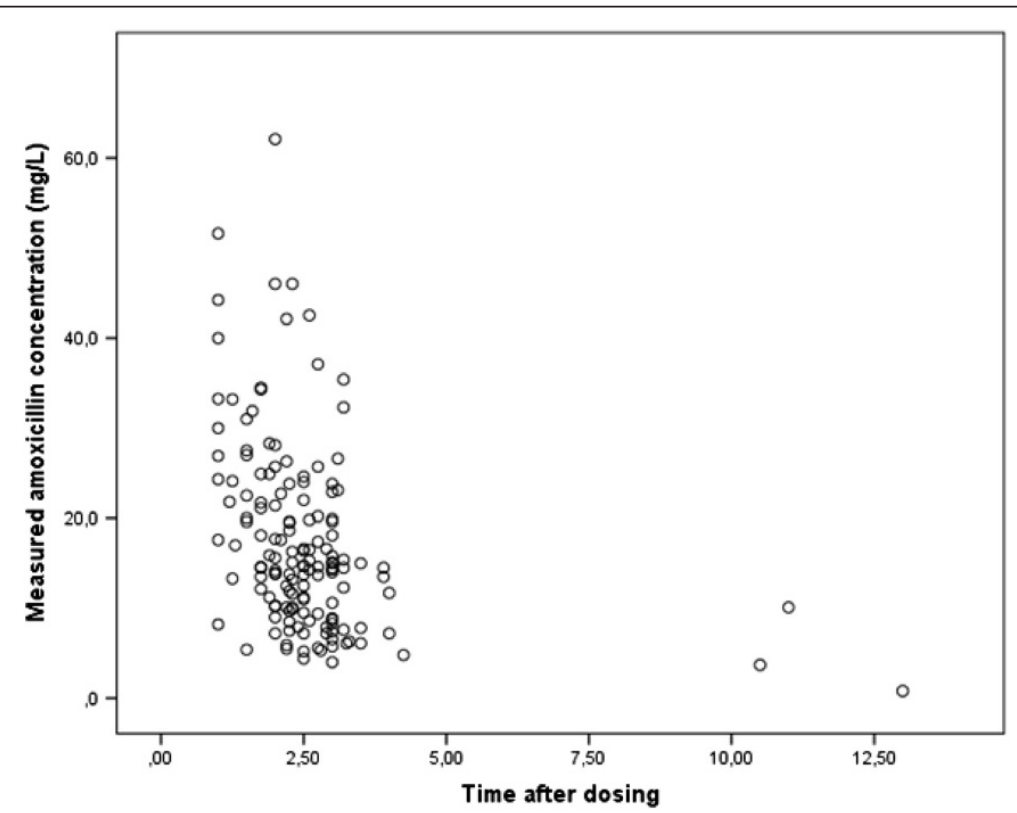

Figure 1 The measured concentrations of amoxicillin plotted against the time after amoxicillin administration. 


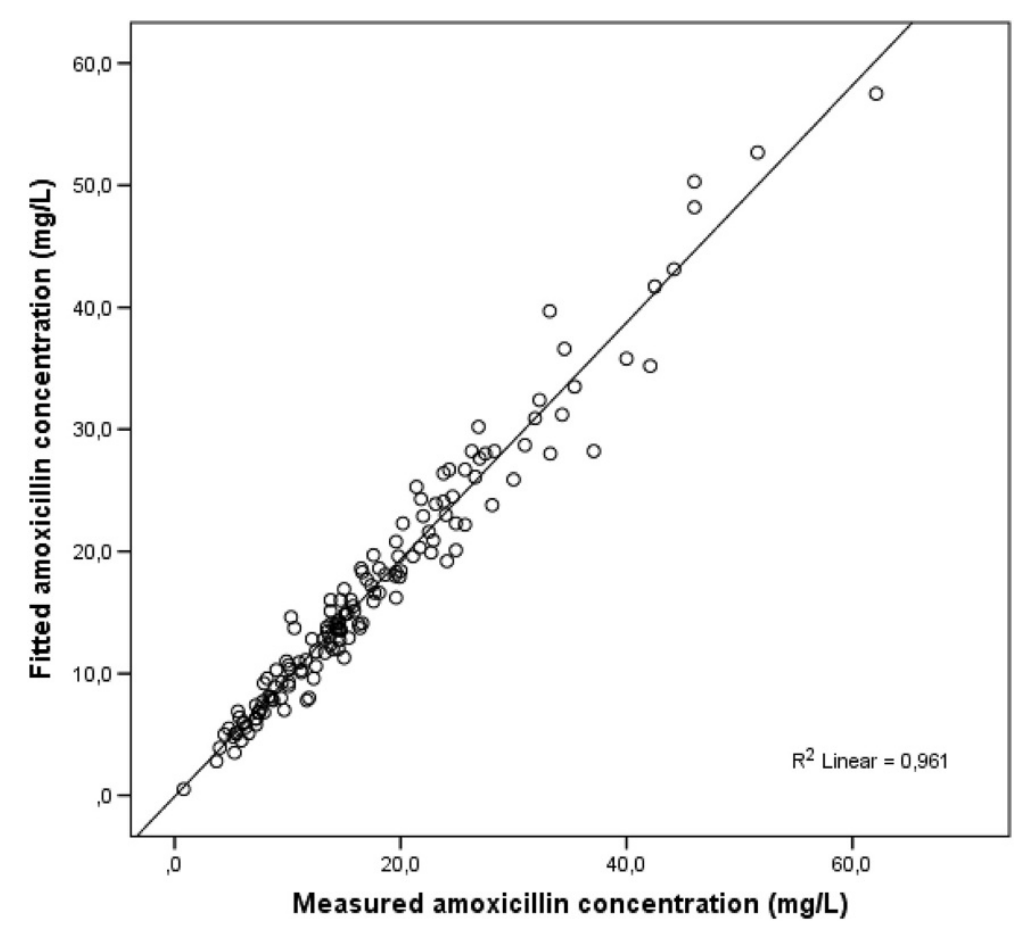

Figure 2 Correlation between amoxicillin actual measured concentration and estimated with maximum a posteriori Bayesian fitting (MW/Pharm 3.60, Mediware, the Netherlands).

\section{Discussion}

In this study, we demonstrate that the efficacy target of $40 \% \mathrm{~T}>\mathrm{MIC}$ for amoxicillin/clavulanic acid was reached in $93 \%$ of the patients tested when the MIC was $4 \mathrm{mg} / \mathrm{L}$ and only in $65 \%$ of the patients tested when the MIC was $8 \mathrm{mg} / \mathrm{L}$. In the EUCAST and CLSI criteria Enterobacteriaceae are considered to be susceptible for amoxicillin/clavulanic acid with bacterial $\mathrm{MIC} \leq 8 \mathrm{mg} / \mathrm{L}$ [11]. High bacterial MICs for amoxicillin/clavulanic acid are an increasing problem in the Netherlands and in Europe
$[19,20]$. To prevent treatment failure for individual patients and to prevent development of antibiotic resistance on population level, increasing the standard dose of amoxicillin/clavulanic acid seems warranted. Dosing simulation showed that increasing the standard dose of amoxicillin/clavulanic acid to 6 times a day 1000/200 mg increased the number of patients reaching $40 \% \mathrm{~T}>\mathrm{MIC}$ to $100 \%$ for bacterial $\mathrm{MIC} \leq 4$ and to $95 \%$ with bacterial $\mathrm{MIC} \leq 8$. Continuous iv dosing is an alternative for frequent dosing of time dependent $\beta$-lactam antibiotics.

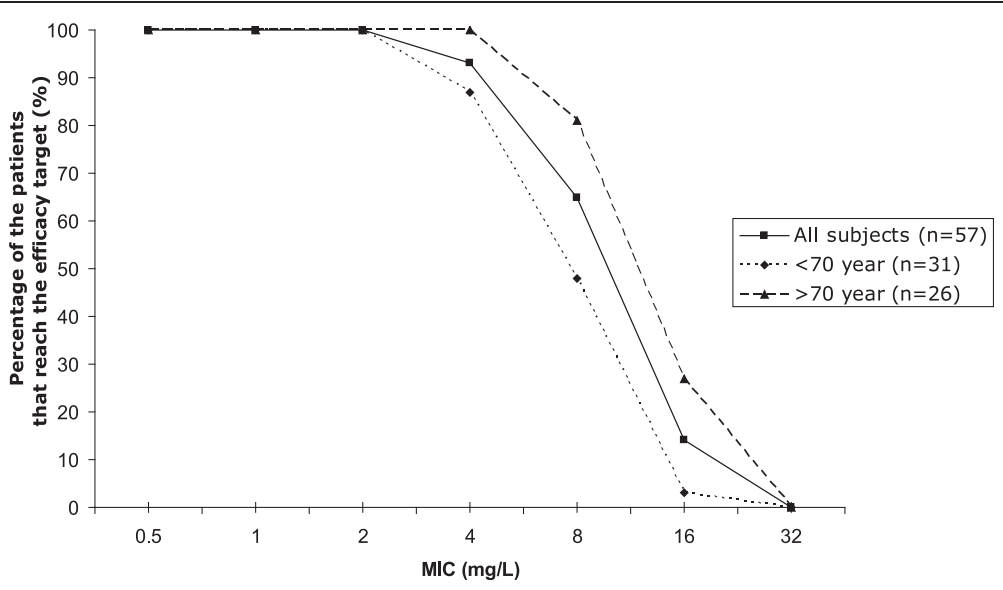

Figure 3 The percentage of patients that reach the $40 \%$ T > MIC for different age categories at different MICs. 
Table 3 Univariate Pearson correlation coefficients between amoxicillin/clavulanic acid, CLam/W and predictors used in this study

\begin{tabular}{lll}
\hline & \multicolumn{2}{c}{ Univariate CLam/W } \\
\cline { 2 - 3 } & $\mathbf{R}$ & $\boldsymbol{P}$-value \\
\hline Creatinine & -0.584 & $<0.001$ \\
Age & -0.476 & $<0.001$ \\
Gender & -0.034 & 0.812 \\
V/W & -0.608 & $<0.001$ \\
40\%T $>$ MIC & -0.424 & 0.025 \\
\hline
\end{tabular}

CLam/W, amoxicillin/clavulanic acid clearance corrected for bodyweight. $\mathrm{V} / \mathrm{W}$, amoxicillin/clavulanic acid volume of distribution corrected for bodyweight.

Unfortunately, amoxicillin is not very suitable for continuous iv dosing, because of the instability of amoxicillin at room temperature. Therefore, we suggest increasing the dose of amoxicillin/clavulanic acid to 6 times a day in patients with severe Enterobacteriaceae sepsis or intraabdominal infection.

In general amoxicillin/clavulanic acid is well tolerated. The most frequent adverse drug events are diarrhoea, nausea and vomiting. However, amoxicillin/clavulanic acid is also associated with liver injury, which is estimated to occur from 1 to 1.7 per 10.000 users [21,22]. Clavulanic acid seems to be responsible for the adverse drug reaction, since amoxicillin alone is rarely associated with liver injury and causes less gastrointestinal problems than the combination preparation [23-25]. In vitro pharmacodynamic studies demonstrate that low dose of clavulanic acid suffice and the $\beta$-lactamase inhibition of clavulanic acid lasts for 8-12 hours [26,27]. Therefore, increasing the standard dose of amoxicillin/clavulanic acid of 4 times a day $1000 / 200 \mathrm{mg}$ iv with amoxicillin twice daily $1000 \mathrm{mg}$ iv may be a safe and effective alternative.

CLam/W is correlated with CLcr and the amoxicillin dose is adjusted with to the CLcr. However, other covariates also influence the CLam/W. CLam/W was significantly correlated with age. However, age and creatinine were not correlated to each other, meaning that elderly patients can have both a normal creatinine and a decreased CLam/W. Therefore, the correlation of age with CLam/W seems independent of the creatinine. Furthermore, the 4 patients that did not reach the efficacy target with bacterial $\mathrm{MIC}=4$ were all young patients with excellent clearance. Our measured attainment results are lower than those calculated attainment results in the EUCAST rationale document, in which Monte Carlo simulations were used to calculate the target attainment rates $(40 \% \mathrm{~T}>\mathrm{MIC})$ of different dosing regimens (from $500 \mathrm{mg} 3$ times a day to $2 \mathrm{~g} 4$ times a day) for different bacterial MICs $(0.5-32 \mathrm{mg} / \mathrm{L})$ [11]. The target attainment in the EUCAST rationale document for the standard dose $(1000 / 200 \mathrm{mg} 4$ times a day) is $100 \%$ at bacterial $\mathrm{MIC} \leq 4$ and $75 \%$ with bacterial $\mathrm{MIC} \leq 8 \mathrm{mg} / \mathrm{L}[11]$. In our real life blood level determination study, these percentages were $93 \%$ and $65 \%$, respectively. This difference may be explained by the larger interindividual variability of our population and in particular by a group of younger patients with normal renal clearance (CLcr $>60 \mathrm{~mL} / \mathrm{min}$ ). Remarkably, in the EUCAST rationale document, the interindividual variation is extremely small; $t^{1} \frac{2}{2}$ is $1.1( \pm 0.1) h$, versus $t^{1} \frac{2}{2}$ is $1.5( \pm 0.6) h$ in our study. The higher $t^{1 / 2}$ in our study may be due to the high mean age of our population.

No significant associations have been found between the target $40 \% \mathrm{~T}>\mathrm{MIC}$ and clinical outcome. As expected, our study population was too small and too heterogeneous. A large number of patients are needed to draw

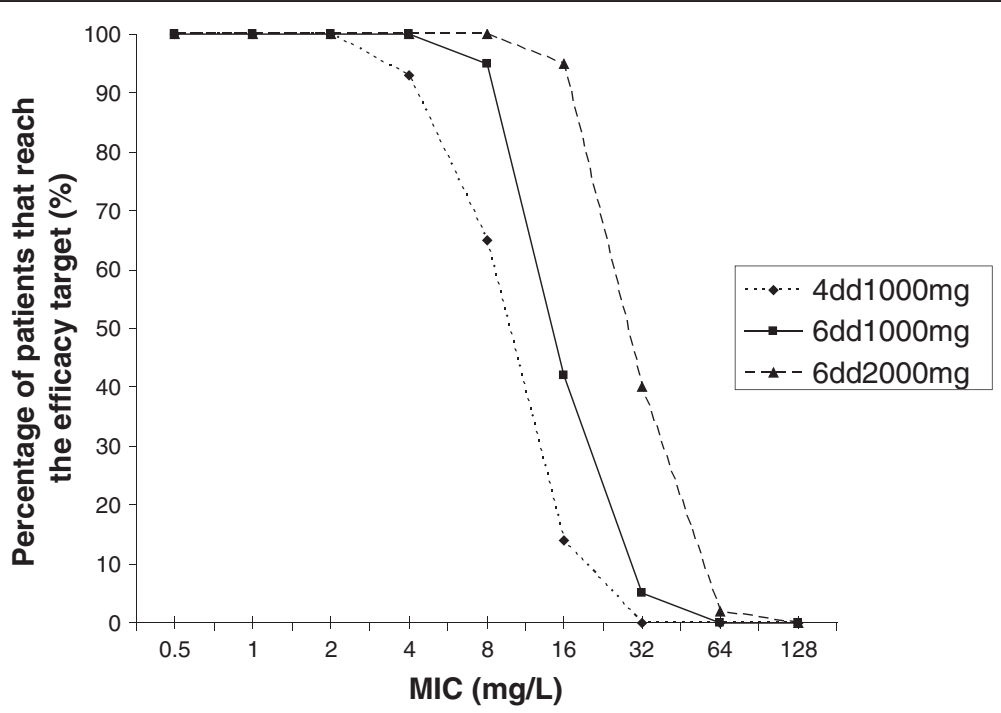

Figure 4 Calculated percentage of patients with $40 \%$ T > MIC at different MICs for increasing amoxicillin dosages. 
conclusions for this endpoint. Moreover, in an in vitro study, ceftazidim has been shown to be maximally effective when $40 \% \mathrm{~T}>\mathrm{MIC}$ was reached for concentrations four times the MIC or higher [28]. In our study, the target of $40 \% \mathrm{~T}>4 \times \mathrm{MIC}$ was only reached in $100 \%$ for low MICs $(\leq 1 \mathrm{mg} / \mathrm{L})$, but never for bacterial MICs of 4 and $8 \mathrm{mg} / \mathrm{L}$. Our study was not designed to isolate a large number of bacterial MICs and therefore only a limited number of clinical bacterial MICs were available in our study. Taken together a large clinical PK/PD study of amoxicillin/clavulanic acid is needed with microbiological and clinical cure endpoints to establish the association between clinical endpoints and the efficacy target $40 \% \mathrm{~T}>$ MIC.

\section{Conclusions}

The current standard dose of amoxicillin/clavulanic acid 4 times a day $1000 / 200 \mathrm{mg}$ iv is too low to reach the $40 \% \mathrm{~T}$ > MIC for bacterial MIC of $8 \mathrm{mg} / \mathrm{L}$ in a high percentage of patients. To prevent treatment failure for individual patients and to prevent development of antibiotic resistance on population level, we suggest increasing the standard dose of amoxicillin/clavulanic acid to 6 times a day in patients with an Enterobacteriaceae sepsis or intra-abdominal infection.

\section{Abbreviations}

AST: Antibiotic susceptibility testing; CLam/W: Amoxicillin clearance corrected for bodyweight; CLcr: Creatinine clearance; CRP: C-reactive protein;

ID: Identification of the causative bacterium; iv: Intravenous; MAP: Maximum a posteriori; MIC: Minimal inhibition concentration; MUMC: Maastricht University Medical Centre; PK: Pharmacokinetic; RP-HPLC: Reverse phase high pressure liquid chromatography; SD: Standard deviation; T: Time; VM: Volume of distribution corrected for bodyweight.

\section{Competing interests}

The authors declare that they have no competing interests.

\section{Authors' contributions}

$\mathrm{MH}, \mathrm{TH}$ and $\mathrm{LS}$ carried out the data analysis. CB, LS, CN an AV participated in design of the study. MH, TH, LS, CN, CB and AV drafted the manuscript. All authors have read and approved the final version manuscript.

\section{Acknowledgements}

We acknowledge the support provided by department of Clinical Chemistry and the Pharmacy laboratory. We also acknowledge the assistance of Dr. Bekers and the excellent technical assistance of Jeroen Welzen and Pauline Vinken. This work was supported by Care and Public Health Research Institute (CAPHRI), Maastricht, the Netherlands and the Medical University Centre Maastricht, the Netherlands.

\section{Author details}

${ }^{1}$ Department of Medical Microbiology, Maastricht University Medical Centre, Maastricht, the Netherlands. ${ }^{2}$ Department of Clinical Pharmacy, Maastricht University Medical Centre, Maastricht, the Netherlands. ${ }^{3}$ Care and Public Health Research Institute (CAPHRI), Maastricht, the Netherlands. ${ }^{4}$ Department of Internal Medicine, Erasmus Medical Centre, Rotterdam, the Netherlands, ${ }^{5}$ Present address: Maastricht University Medical Centre, P. Debyelaan 25, PO Box 58006202 AZ Maastricht, the Netherlands.

Received: 24 March 2014 Accepted: 14 July 2014

Published: 21 July 2014

\section{References}

1. Craig WA: Antimicrobial resistance issues of the future. Diagn Microbiol Infect Dis 1996, 25(4):213-217.

2. Vogelman B, Craig WA: Kinetics of antimicrobial activity. J Pediatr 1986, 108(5 Pt 2):835-840.

3. Craig WA: Interrelationship between pharmacokinetics and pharmacodynamics in determining dosage regimens for broad-spectrum cephalosporins. Diagn Microbiol Infect Dis 1995, 22(1-2):89-96.

4. Adam D, de Visser I, Koeppe P: Pharmacokinetics of amoxicillin and clavulanic acid administered alone and in combination. Antimicrob Agents Chemother 1982, 22(3):353-357.

5. Sjovall J, Alvan G, Huitfeldt B: Intra- and inter-individual variation in pharmacokinetics of intravenously infused amoxycillin and ampicillin to elderly volunteers. Br J Clin Pharmacol 1986, 21(2):171-181.

6. Matsuura M, Nakazawa H, Hashimoto T, Mitsuhashi S: Combined antibacterial activity of amoxicillin with clavulanic acid against ampicillin-resistant strains. Antimicrob Agents Chemother 1980, 17(6):908-911.

7. van Zanten AR, Polderman KH, van Geijlswijk IM, van der Meer GY, Schouten MA, Girbes AR: Ciprofloxacin pharmacokinetics in critically ill patients: a prospective cohort study. J Crit Care 2008, 23(3):422-430.

8. Haeseker M, Stolk L, Nieman F, Hoebe C, Neef C, Bruggeman C, Verbon A: The ciprofloxacin target AUC : MIC ratio is not reached in hospitalized patients with the recommended dosing regimens. $\mathrm{Br} J$ Clin Pharmacol 2013, 75(1):180-185.

9. Aubert G, Carricajo A, Coudrot M, Guyomarc'h S, Auboyer C, Zeni F: Prospective determination of serum ceftazidime concentrations in intensive care units. Ther Drug Monit 2010, 32(4):517-519.

10. Rea RS, Capitano B, Bies R, Bigos KL, Smith R, Lee H: Suboptimal aminoglycoside dosing in critically ill patients. Ther Drug Monit 2008, 30(6):674-681

11. EUCAST: Amoxicillin Rationale for the EUCAST clinical breakpoints. www eucast.org/documents/rd/.

12. Nederlands Trial Register. Antibiotic blood levels elderly. http://www. trialregister.nl/trialreg/admin/rctview.asp?TC=1725.

13. FDA: Guidance for Industry. Bioanalytical Method Validation, U.S. Department of Health and Food Science. Rockville, MD, USA: Food and Drug Administration (FDA); 2001.

14. Martinez MN, Riviere JE: Review of the 1993 veterinary drug bioequivalence workshop, held on march 29-31, 1993, in Rockville, Maryland. J Vet Pharmacol Ther 1994, 17(2):85-119.

15. Pullen J, Stolk LM, Neef C, Zimmermann LJ: Microanalysis of amoxicillin, flucloxacillin, and rifampicin in neonatal plasma. Biomed Chromatogr 2007, 21(12):1259-1265.

16. Proost JH, Meijer DK: MW/Pharm, an integrated software package for drug dosage regimen calculation and therapeutic drug monitoring. Comput Biol Med 1992, 22(3):155-163.

17. van der Meer AF, Marcus MA, Touw DJ, Proost JH, Neef C: Optimal sampling strategy development methodology using maximum a posteriori Bayesian estimation. Ther Drug Monit 2011, 33(2):133-146.

18. Turnidge JD: The pharmacodynamics of beta-lactams. Clin Infect Dis 1998, 27(1):10-22.

19. Blaettler L, Mertz D, Frei R, Elzi L, Widmer AF, Battegay M, Fluckiger U: Secular trend and risk factors for antimicrobial resistance in Escherichia coli isolates in Switzerland 1997-2007. Infection 2009, 37(6):534-539.

20. SWAB: Consumption of antimicrobial agents and antimicrobial resistance among medically important bacteria in the Netherlands. http://www. swab.nl/swab/cms3.nsf/viewdoc/20BCD3983B5C390AC12575850031D33D.

21. de Abajo FJ, Montero D, Madurga M, Garcia Rodriguez LA: Acute and clinically relevant drug-induced liver injury: a population based casecontrol study. Br J Clin Pharmacol 2004, 58(1):71-80.

22. Garcia Rodriguez LA, Stricker BH, Zimmerman HJ: Risk of acute liver injury associated with the combination of amoxicillin and clavulanic acid. Arch Intern Med 1996, 156(12):1327-1332.

23. Salvo F, Polimeni G, Moretti U, Conforti A, Leone R, Leoni O, Motola D, Dusi G, Caputi AP: Adverse drug reactions related to amoxicillin alone and in association with clavulanic acid: data from spontaneous reporting in Italy. J Antimicrob Chemother 2007, 60(1):121-126.

24. Bolzan H, Spatola J, Castelletto R, Curciarello J: Intrahepatic cholestasis induced by amoxicillin alone. Gastroenterol Hepatol 2000, 23(5):237-239.

25. Schwarze C, Schmitz V, Fischer HP, Sauerbruch T, Spengler U: Vanishing bile duct syndrome associated with elevated pancreatic enzymes after 
short-term administration of amoxicillin. Eur J Gastroenterol Hepatol 2002 14(11):1275-1277.

26. Aguilar L, Martin M, Balcabao IP, Gomez-Lus ML, Dal-Re R, Prieto J: In vitro assessment of the effect of clavulanic acid at concentrations achieved in human serum on the bactericidal activity of amoxicillin at physiological concentrations against Staphylococcus aureus: implications for dosage regimens. Antimicrob Agents Chemother 1997, 41(6):1403-1405.

27. Cooper CE, Slocombe B, White AR: Effect of low concentrations of clavulanic acid on the in-vitro activity of amoxycillin against betalactamase-producing Branhamella catarrhalis and Haemophilus influenzae. J Antimicrob Chemother 1990, 26(3):371-380.

28. Mouton JW, Punt N, Vinks AA: Concentration-effect relationship of ceftazidime explains why the time above the MIC is 40 percent for a static effect in vivo. Antimicrob Agents Chemother 2007, 51(9):3449-3451.

doi:10.1186/2050-6511-15-38

Cite this article as: Haeseker et al: Is the standard dose of amoxicillinclavulanic acid sufficient? BMC Pharmacology and Toxicology 2014 15:38.

\section{Submit your next manuscript to BioMed Central and take full advantage of:}

- Convenient online submission

- Thorough peer review

- No space constraints or color figure charges

- Immediate publication on acceptance

- Inclusion in PubMed, CAS, Scopus and Google Scholar

- Research which is freely available for redistribution 